\title{
ECG-Based Detection of Body Position Changes in Ischemia Monitoring
}

\author{
José García*, Magnus Åström, Javier Mendive, Pablo Laguna, and Leif Sörnmo, Member, IEEE
}

\begin{abstract}
The purpose of this paper is to analyze and detect changes in body position (BPC) during electrocardiogram (ECG) recording. These changes are often manifested as shifts in the electrical axis and may be misclassified as ischemic changes during ambulatory monitoring. We investigate two ECG signal processing methods for detecting BPCs. Different schemes for feature extraction are used (spatial and scalar), while preprocessing, trend postprocessing and detection are identical. The spatial approach is based on VCG loop rotation angles and the scalar approach is based on the Karhunen-Loève transform (KLT) coefficients. The methods are evaluated on two different databases: a database with annotated BPCs and the STAFF III database with recordings from rest and during angioplasty-induced ischemia but not including BPCs. The angle-based detector results in performance values of detection probability $P_{D}=95 \%$, false alarm probability $P_{F}=3 \%$ in the BPC database and false alarm rate in the STAFF III database in control ECGs during rest $R_{F}(c)=2 \mathrm{~h}^{-1}$ (episodes per hour) and in ischemia recordings during angioplasty $R_{F}(a)=7 \mathrm{~h}^{-1}$, whereas the KLT-based detector produces values of $P_{D}=89 \%, P_{F}=3 \%, R_{F}(c)=4 \mathrm{~h}^{-1}$, and $R_{F}(a)=11 \mathrm{~h}^{-1}$, respectively. Including information on noise level in the detection process to reduce the number of false alarms, performance values of $P_{D} \simeq 90 \%, P_{F} \simeq 1 \%, R_{F}(c) \simeq 1 \mathrm{~h}^{-1}$ and $R_{F}(a) \simeq 2 \mathrm{~h}^{-1}$ are obtained with both methods. It is concluded that reliable detection of BPCs may be achieved using the ECG signal and should work in parallel to ischemia detectors.
\end{abstract}

Index Terms-Alarms, body position changes, detection, ECG, ischemia.

\section{INTRODUCTION}

M YOCARDIAL ischemia is usually detected in the surface electrocardiogram (ECG) from the repolarization period (ST-T complex). Ischemia may be detected from abnormal amplitudes change measured from the ST-T complex. However, changes in the ST segment or ST-T complex are not always due to ischemia, but to other factors as body position

Manuscript received May 30, 2002; revised September 16, 2002. This work was supported by projects TIC2001-2167-C02:02 and TIC2001-2481 from Comisión Interministerial de Ciencia y Tecnología (CICYT) and Fondos Europeos de Desarrollo (FEDER), FIS G03/117 from Fondo de Investigación Sanitaria, CONSID P075/2001 from Gobierno de Aragón, and grants from the Swedish National Board for Technical Development (VINNOVA). Asterisk indicates corresponding author.

*J. García is with the Communications Technologies Group (GTC) Aragón Institute for Engineering Research (I3A) Polytechnic Center, University of Zaragoza, Maria de Luna 1., 50018 Zaragoza, Spain (e-mail: jogarmo@ posta.unizar.es).

M. Åström and L. Sörnmo are with the Signal Processing Group, Department of Electroscience, Lund University, SE-221 00 Lund, Sweden.

J. Mendive and P. Laguna are with the Communications Technologies Group (GTC) Aragón Institute for Engineering Research (I3A) Polytechnic Center, University of Zaragoza, 50018 Zaragoza, Spain.

Digital Object Identifier 10.1109/TBME.2003.812208

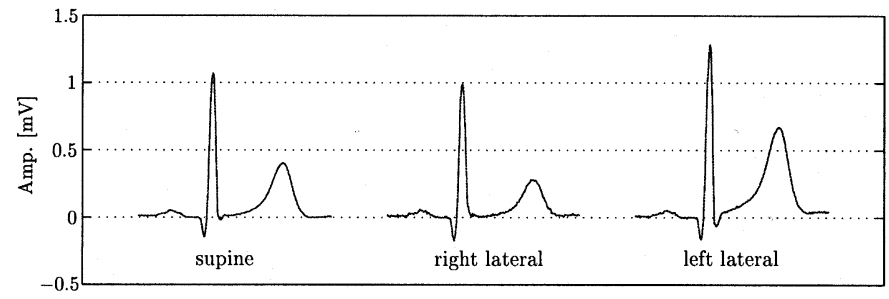

Fig. 1. Beat morphologies for three positions recorded in lead X from the same individual. The differences found in ST segment level and T wave amplitude between right and left lateral positions are of $83 \mu \mathrm{V}$ and $405 \mu \mathrm{V}$, respectively.

changes (BPC), drug effects, resting potential conditions, etc. BPCs effects on the ECG are manifested as transient changes and also as ECG waveform variations that remain while the new body position is maintained [1], [2]. It is well-known that BPCs are often misclassified by monitoring equipment used in the intensive care unit resulting in false ischemia alarms. At present, software is rarely included in such devices to cancel the false alarms or, at least, to inform the physician that the observed event is likely due to a BPC and not related to an ischemic episode. The large morphologic changes caused by changes from one body position to another are shown in Fig. 1. In this example, the differences in ST segment level and T wave amplitude between right and left lateral positions, are of $83 \mu \mathrm{V}$ and $405 \mu \mathrm{V}$, respectively.

Previous work concerning BPC is descriptive in nature and presents results on how various ECG measurements are affected by changes in body position; methodological development regarding the BPC detection problem is lacking. In [1], the standard 12-lead ECG and the derived 12-lead ECG (Dower's EASI lead system) are compared studying QRS and ST variations for different positional changes. It was concluded that such changes affect all ECG measurements although those related to QRS morphology are more sensitive. Further, the 12-lead system seems to be more sensitive to changes than the derived lead system is. Changes in QRS waveform and ST-T complex during BPCs were also studied in [2], where it was found that the effects on the ST segment were usually small. A recent paper deals with BPCs in relation to ischemia monitoring [3]. In that paper, alterations are studied in terms of vector magnitude differences of the QRS complex and changes of the ST-T complex. The results show that both measures are rather sensitive to BPCs, especially to changes to the left lateral position. The conclusion is that automated monitoring algorithms have a limited value as long as these are not used in combination with a BPC detector. In [4], ECG variations caused by BPCs and electrode placement during ambulatory 
TABLE I

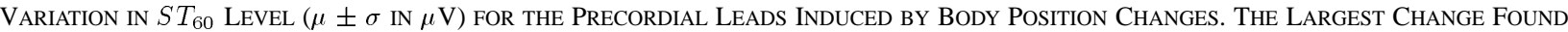
(AND THE CORRESPONDING LeAd) FOR EACH Kind of Positional Change is Also Shown (MAX)

\begin{tabular}{cccccccc}
\hline$B P C$ & $V 1$ & $V 2$ & $V 3$ & $V 4$ & $V 5$ & $V 6$ & Max \\
$S \rightarrow R$ & $32 \pm 28$ & $-38 \pm 30$ & $-7 \pm 32$ & $8 \pm 19$ & $0 \pm 7$ & $0 \pm 6$ & -126 (V2) \\
$R \rightarrow S$ & $-33 \pm 27$ & $33 \pm 27$ & $7 \pm 26$ & $-6 \pm 21$ & $2 \pm 6$ & $3 \pm 5$ & -117 (V4) \\
$S \rightarrow L$ & $-13 \pm 15$ & $-44 \pm 41$ & $14 \pm 50$ & $34 \pm 26$ & $18 \pm 22$ & $8 \pm 13$ & -177 (V3) \\
$L \rightarrow S$ & $12 \pm 14$ & $51 \pm 38$ & $-10 \pm 41$ & $-29 \pm 23$ & $-16 \pm 21$ & $-8 \pm 13$ & $+188(\mathrm{~V} 2)$ \\
\hline
\end{tabular}

TABLE II

VARiation in T WaVe Amplitude $(\mu \pm \sigma$ IN $\mu$ V) For the Precordial LeAds Induced By Body Position Changes. The LaRgest Change Found (AND THE CORRESPONDING LEAD) FOR EACH Kind of POSITIONAL CHANGE IS Also SHOWn (MAX)

\begin{tabular}{cccccccc}
\hline$B P C$ & $V 1$ & $V 2$ & $V 3$ & $V 4$ & $V 5$ & $V 6$ & $M a x$ \\
$S \rightarrow R$ & $119 \pm 91$ & $-97 \pm 106$ & $-65 \pm 94$ & $-15 \pm 76$ & $-38 \pm 48$ & $-30 \pm 29$ & $+398(\mathrm{~V} 2)$ \\
$R \rightarrow S$ & $-115 \pm 87$ & $81 \pm 98$ & $46 \pm 81$ & $5 \pm 69$ & $22 \pm 40$ & $19 \pm 25$ & $-400(\mathrm{~V} 1)$ \\
$S \rightarrow L$ & $-58 \pm 71$ & $-186 \pm 160$ & $-71 \pm 132$ & $78 \pm 160$ & $194 \pm 137$ & $160 \pm 83$ & +686 (V5) \\
$L \rightarrow S$ & $51 \pm 63$ & $199 \pm 141$ & $90 \pm 115$ & $-52 \pm 148$ & $-166 \pm 127$ & $-139 \pm 82$ & $+597(\mathrm{~V} 1)$ \\
\hline
\end{tabular}

monitoring were studied. They analyzed these sources of ECG variations to correct them by reconstructing the signals.

One of the first papers published on the BPC detection problem was by Jager and coworkers who explored the use of Karhunen-Loève transform (KLT) for detection of ischemic ST changes and nonischemic episodes due to, e.g., body position changes [5]. They developed a recognition algorithm that studied the feature vectors evolution in the KLT space for both the ST segment and the QRS complex. Their hypothesis was that during axis shifts the QRS parameters change rapidly (generally over a period of half a minute) generating more than one cluster but not during ST episodes in which case the QRS complexes do not show significant changes. Analysing the pattern of QRS and ST parameters changes, they were able to distinguish between ischemic and nonischemic episodes in the European ST-T Database [6].

In this paper, we compare two different approaches to the BPC detection problem (spatial approach (VCG) and scalar approach (KLT)). Both methods are tested on two different databases, described in Section II. The two methods, the preprocessing and the detection scheme are explained in Section III. Finally, the results and discussion are presented in Section IV and Section V, respectively.

\section{REFERENCE MATERIAL}

The evaluation of a BPC detector needs to be done with reference to different aspects since it will work in parallel to an ischemia detector. First, it is necessary to evaluate the ability of the system to detect BPCs and second, whether or not it confuses BPCs with ischemic episodes. Therefore, two databases were considered with standard 12-lead ECGs recorded with a sampling rate of $1 \mathrm{kHz}$ and an amplitude resolution of $0.6 \mu \mathrm{V}$ (equipment by Siemens-Elema AB, Solna, Sweden).

\section{A. Healthy Individuals Doing BPCs-BPC Database}

The BPC database was recorded following the next protocol: supine — right side — supine — left side and so on. The complete sequence was repeated five times with a duration of 1 min per $\mathrm{BPC}$ in order to give more reliable statistical results. The interval between the changes ( $1 \mathrm{~min}$ ) was chosen in order to allow muscular activity and other artifacts to decay before the next BPC was initiated. Twenty healthy individuals were included in the BPC database (11 males/9 females, $32 \pm 9$ years old).

In order to establish the characteristics of the BPC database, a summary of its properties is presented in Tables I and II. The influence of BPCs on the cardiac cycle was analyzed, looking at changes induced in $S T_{60}$ level and $\mathrm{T}$ wave amplitude, which may yield false ischemia detections. The $S T_{60}$ level and T wave amplitude were estimated with reference to the isoelectric level, during the time intervals without BPCs (excluding beats from 5 $\mathrm{s}$ before each BPC until $10 \mathrm{~s}$ after). One average was obtained for each resting phase. Then all BPCs were characterized leadwise by the difference between two consecutive mean values of $S T_{60}$ level and $\mathrm{T}$ wave amplitude. In Tables I and II, the main results of variations (average values for all the BPCs in all the patients), classified by the different pattern of changes $(S \rightarrow R$ : supine to right side, $L \rightarrow S$ : left side to supine and so on), are presented, showing a significant increase/decrease of $S T_{60}$ level and $\mathrm{T}$ wave amplitude values provoked by the BPCs. One result of this study was that BPCs were usually larger when related to the left-lying body position, in accordance with [3]. Both $S T_{60}$ level and T wave amplitude were affected by BPCs with large variance between individuals. It was possible to find $S T_{60}$ level deviations of up to $180 \mu \mathrm{V}$ and of more than $600 \mu \mathrm{V}$ for T wave amplitude.

This preliminary analysis shows clearly that BPCs can induce significant changes, sometimes of the same order as the threshold in an ischemia detection system (ischemic episodes are commonly defined as deviations of more than $100 \mu \mathrm{V}$ for the ST segment level or $200 \mu \mathrm{V}$ for the $\mathrm{T}$ wave amplitude [6]). Using these thresholds in an ischemia detector, $43 \%$ of the annotated BPCs would be considered as ischemic changes mostly due to erroneous detections derived from the $\mathrm{T}$ wave.

\section{B. Patients Undergoing PTCA-Staff III Database}

The second database contains severe, induced ischemic events. The study group consisted of 83 patients ( 55 males $/ 28$ females, $61 \pm 12$ years old) receiving elective percutaneous transluminal coronary angioplasty (PTCA), i.e., a balloon was 


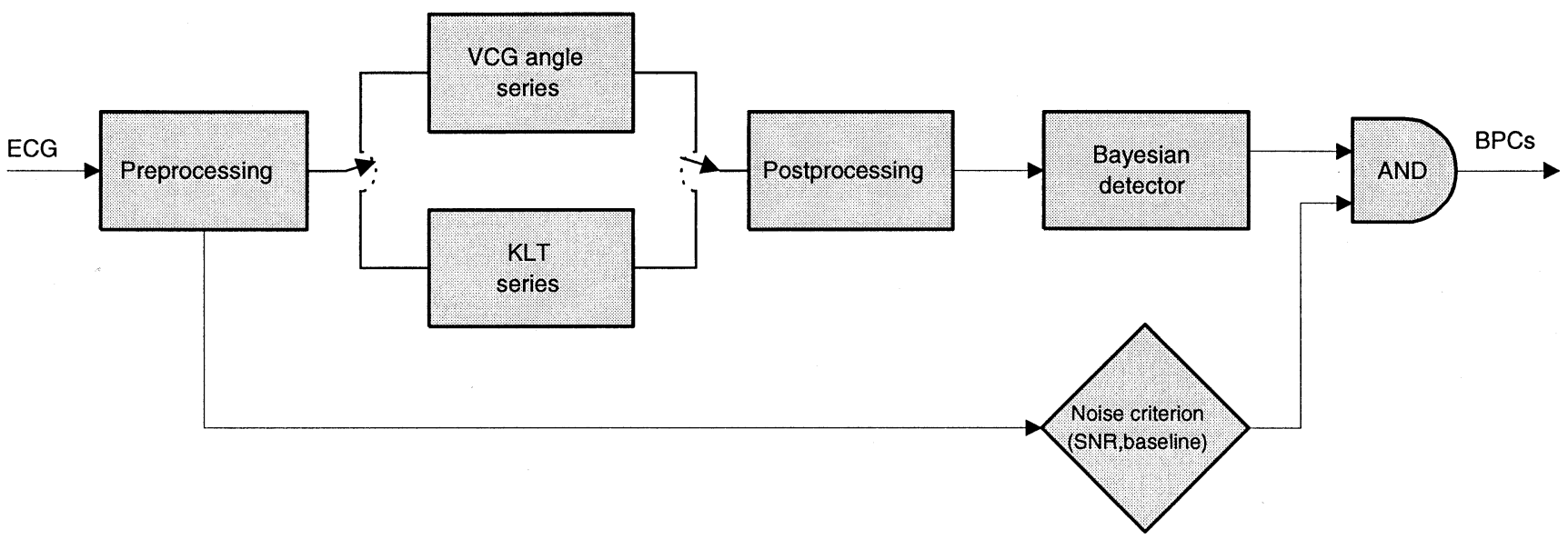

Fig. 2. General scheme for BPC detection. One of the two methods (either VCG angle or KLT series) can be selected.

inflated, blocking the blood flow in one of the major coronary arteries. A control ECG recorded during rest prior to the procedure and the ECG recorded during angioplasty (from the beginning of the recording until PTCA ended) were considered for each patient. In this paper, the recordings obtained during control and angioplasty are denoted with subscripts $c$ and $a$, respectively. A more extensive description of the STAFF III database is found in [7], [8]. It is assumed that no BPCs occurred during the recording of this data (highly unlikely that any of the patients moved during surgery).

\section{METHODS}

The BPC detector mainly consists of feature extraction followed by decision making. The following two different feature extraction methods have been compared:

- a spatial approach based on loop rotation angles;

- a scalar approach based on the KLT coefficients.

Both approaches make use of identical preprocessing and postprocessing and detection scheme, see Fig. 2. In parallel to the above algorithms, an extra stage including information on noise level in the detection process to reduce the number of false alarms was added.

\section{A. Preprocessing}

The preprocessing of the recorded ECG signal consists of the following stages: baseline filtering, synthesis of vectorcardiographic (VCG) leads, QRS detection and rejection of beats with low signal-to-noise ratio (SNR). The synthesized VCG signals are obtained from the standard 12-lead ECG using the inverse Dower matrix [9]. The baseline wander is removed by using a time varying linear high-pass filter (third-order Butterworth) with a normalized cutoff frequency depending on the heart rate beat frequency [10]. The QRS detection is based on an envelope signal of the ECG and the decision rule is applied to it by using a multistage process [11].

Beats with poor signal quality, e.g., due to myoelectric noise, are rejected since these otherwise would result in unrealistic trend fluctuations. The problem of removing low SNR beats is complicated by the fact that different body positions as well as different recordings may have different SNR levels. In order to solve that, an exponentially updated SNR threshold is used so that it is possible to eliminate noisy beats for a shorter period of time and to maintain the ability to detect body position changes at low SNRs. Consecutive beats with baseline isoelectric levels differing in more than $0.6 \mathrm{mV}$ are also discarded.

\section{B. Spatial Approach Based on VCG Angle Series}

The method is based on the observation that a BPC causes a change in the position of the heart and, as a result, the ECG/VCG morphology is altered. One way to quantify such morphologic alterations is to estimate the relative rotation angles of successive VCG loops and then to search for angle signatures which are characteristic of BPCs. The maximum likelihood approach to the estimation of rotation angles, recently presented in [12], is briefly summarized below. The estimation is based on a model in which the observed VCG loop for a beat, $\mathbf{Z}$, is related through rotation, defined by the matrix $\mathbf{Q}$, plus alignment handled by the shift matrix $\mathbf{J}_{\tau}$ and scaling by the amplitude factor $\alpha$, to a "reference" loop $\mathbf{Z}_{R}$ and corrupted by white, zero-mean Gaussian noise

$$
\mathbf{Z}=\alpha \mathbf{Q} \mathbf{Z}_{R} \mathbf{J}_{\tau}+\mathbf{W}
$$

The loops $\mathbf{Z}$ and $\mathbf{Z}_{R}$ are represented by matrices, defined by the three leads $X, Y$ and $Z$ and the number of samples embracing the $\mathrm{QRS}$ complex. The rotation matrix $\mathrm{Q}$ is a $3 \times 3$ orthonormal matrix. The problem of selecting the reference loop $\mathbf{Z}_{R}$ is addressed in [12].

The maximum likelihood criterion for estimating $\mathbf{Q}$ leads to that the Frobenius norm between the original and estimated loops should be minimized with respect to $\mathrm{Q}$. From the resulting estimate $\hat{\mathbf{Q}}$, the rotation angles defining the three different planar rotations are calculated by

$$
\begin{aligned}
& \hat{\varphi}_{Y}=\arcsin \left(\hat{q}_{13}\right) \\
& \hat{\varphi}_{X}=\arcsin \left(\frac{\hat{q}_{12}}{\cos \hat{\varphi}_{Y}}\right) \\
& \hat{\varphi}_{Z}=\arcsin \left(\frac{\hat{q}_{23}}{\cos \hat{\varphi}_{Y}}\right)
\end{aligned}
$$



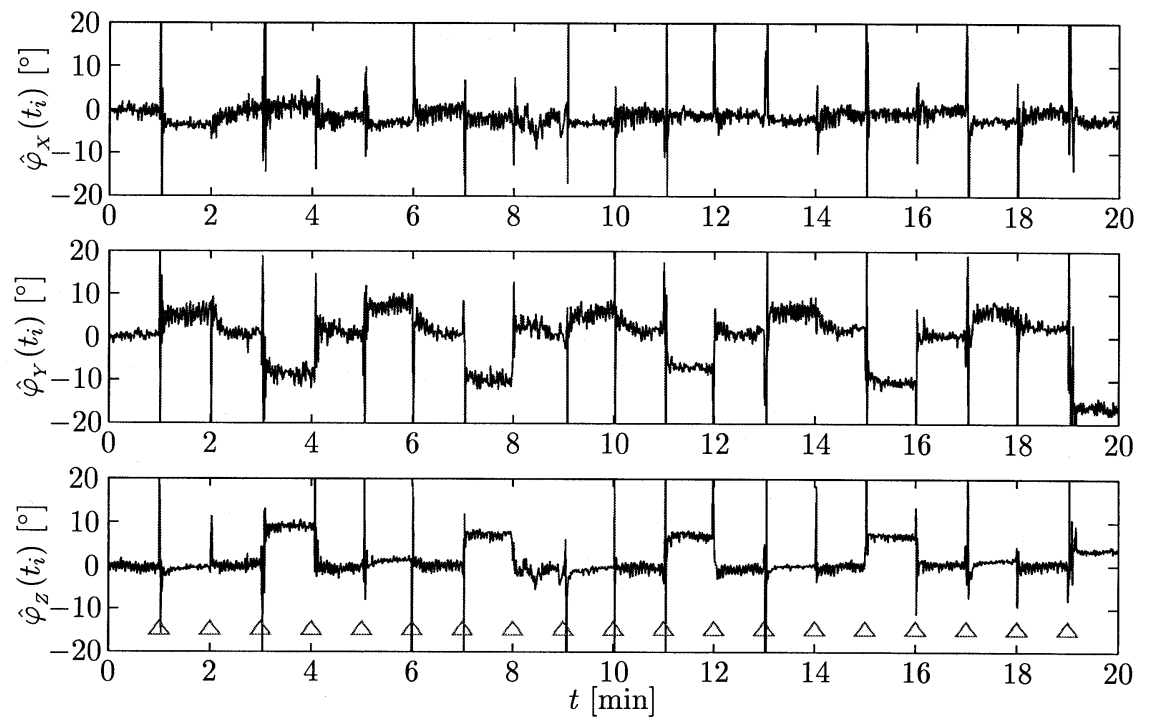

Fig. 3. Example of rotation angle estimates. The instants of the BPCs are denoted with " $\triangle$."

where $\hat{q}_{m n}$ denotes the element of the $m$ th row and $n$th column of $\hat{\mathbf{Q}}$. The estimation procedure is then repeated for each new successive loop of the VCG recording.

An example of the three angle series $\hat{\varphi}_{X}, \hat{\varphi}_{Y}$, and $\hat{\varphi}_{Z}$ is presented in Fig. 3 for a subject included in the BPC database. In this example, changes in the angle series occur every minute corresponding to the time instants of a BPC.

\section{Scalar Approach Based on Karhunen-Loève Coefficients}

The KLT technique applied to different waves of the ECG signal provides a useful tool to estimate their morphologic changes. The KLT is an orthogonal linear transform, optimal in the sense that it concentrates the signal information in a few coefficients [13]. The dynamic beat-to-beat evolution of the signal can be characterized by the coefficient time series. In this paper, the KLT has been applied to the QRS complex and the entire ST-T complex in order to analyze how BPCs affect the ECG signal. The details on how this transform was developed and applied to the ECG segments are described in [14], [15].

In this paper, both the QRS and the ST-T complexes are considered for the detection of body position changes. A combined distance function of the corresponding KLT time series for QRS and ST-T is defined as

$$
\mathcal{F}\left(t_{i}\right)=\lambda_{\mathrm{QRS}} \cdot f_{\mathrm{QRS}}\left(t_{i}\right)+\lambda_{\mathrm{STT}} \cdot f_{\mathrm{STT}}\left(t_{i}\right)
$$

where $\lambda_{\mathrm{QRS}}, \lambda_{\mathrm{STT}}$ represent the weights for the functions and $f_{\mathrm{QRS}}\left(t_{i}\right), f_{\mathrm{STT}}\left(t_{i}\right)$ are the distance functions for each complex at time $t_{i}$. These functions are simply the distance series between each KLT coefficients vector (in which only the first four components are considered) and a mean reference value $(r)$ estimated from the onset of the recording

$$
f\left(t_{i}\right)=\sum_{j=1}^{3}\left(\sum_{k=0}^{3}\left(\alpha_{k}^{j}\left(t_{i}\right)-\alpha_{k}^{j}(r)\right)^{2}\right)^{1 / 2}
$$

with $\alpha_{k}^{j}\left(t_{i}\right)$ being the $k$ th-order KLT coefficient at $t_{i}$ estimated for $j$ th lead beat. The $\alpha_{k}^{j}\left(t_{i}\right)$ coefficient series is estimated using adaptive filtering to remove noise uncorrelated to the signal, thus improving the KLT estimation [14]. A compromise between noise reduction and convergence time is reached using a step-size parameter for the LMS algorithm of $\mu=0.25$, that yields a SNR improvement in the series of more than $6 \mathrm{~dB}$, with a convergence time of one beat [14].

The $\mathcal{F}\left(t_{i}\right)$ function reflects, in a combined way, changes in QRS and ST-T complexes. Although the largest changes during BPCs are usually related to the QRS complex it is also desirable to keep the information of possible changes on the ST-T complex; therefore, values of $\lambda_{\mathrm{QRS}}=0.8$ and $\lambda_{\mathrm{STT}}=0.2$ were experimentally selected as weights. An example of the first KLT coefficient series for both QRS and ST-T complexes in lead $X$ $\left(\alpha_{0}^{X}\right)$ is shown in Fig. 4 (corresponding to the same patient as in Fig. 3). The corresponding KLT distance trend is represented in Fig. 5.

\section{Postprocessing}

Once the rotation angles and KLT trends are obtained, postprocessing is applied to reject outlier values and to resample them at equidistant sample times. A simple but effective method for outlier rejection is the median absolute deviation (MAD) method [16]. The idea of this method is to focus on the median and the median absolute deviation instead of the more commonly used mean and standard deviation. This method is applied to the trends to avoid false BPC detections. An example of the rejection method applied to the KLT distance trend is shown in Fig. 5. The outliers are likely to produce false BPC detections, however, the rejection method eliminates this risk. The outliers are usually due to a sudden drop in SNR.

Finally, the trends are resampled to an even sampling rate. The procedure of resampling is based on linear interpolation. This method is chosen since the introduced delay is minimal and that it is a computationally fast method. The sampling rate after resampling is $f_{s}=2 \mathrm{~Hz}$. 


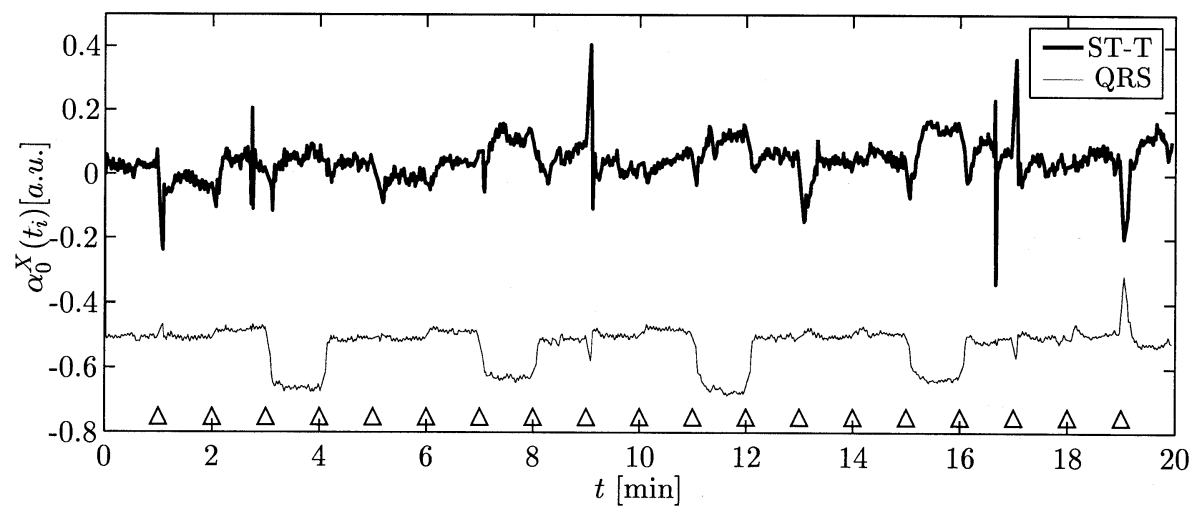

Fig. 4. Example of KLT coefficients for QRS and ST-T complexes (expressed in arbitrary units). The instants of the BPCs are denoted with " $\triangle$ ".

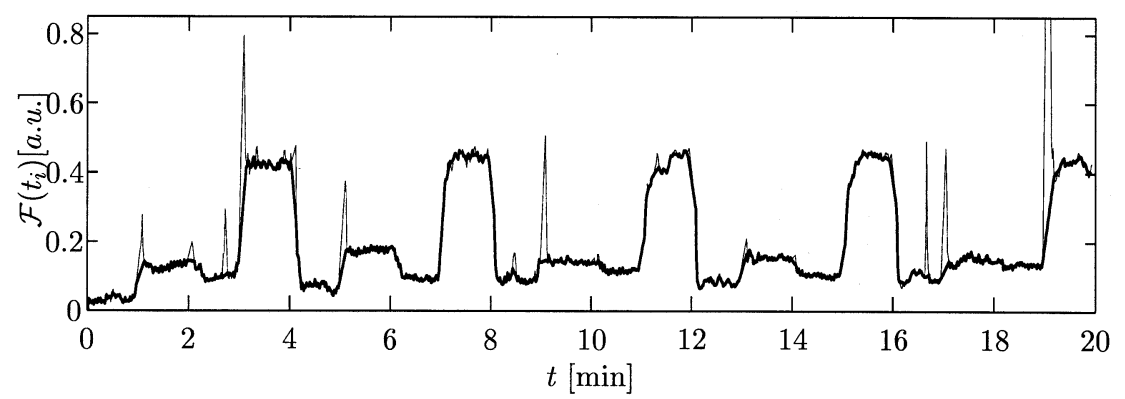

Fig. 5. An example of outlier rejection based on median absolute deviation applied to the KLT distance series: Original trend (thin line) and filtered trend (thick line).

\section{E. Bayesian Detection of BPCs}

A simple statistical model of the basic trend characteristics $\varphi(n)$ is considered with the aim to determine if a BPC has occurred (hypothesis $\mathcal{H}_{1}$ ), or if only noise is present (hypothesis $\mathcal{H}_{0}$ ); this aim is later extended to also handle the detection of multiple BPCs. The model was developed without specific reference to the origin of the time series and is applied equally to trends of KLT coefficients and rotation angles. The onset of the observation interval occurs at the sliding time instant $n=n_{0}$. A $\mathrm{BPC}$ is characterized by the signature $s(n)$ which is disturbed by additive noise $\mathbf{w}(n)$ with mean value $m_{l}$

$$
\begin{aligned}
& \mathcal{H}_{1}: \varphi(n)=\mathbf{a} s\left(n-n_{0}-\theta\right)+\mathbf{w}(n) \\
& \mathcal{H}_{0}: \varphi(n)=\mathbf{w}(n)
\end{aligned}
$$

where $n=n_{0}, \ldots, n_{0}+M-1$ and $\varphi(n)=$ $\left[\begin{array}{lll}\varphi_{1}(n) & \varphi_{2}(n) & \varphi_{3}(n)\end{array}\right]^{T}, \mathbf{w}(n)$ and $\mathbf{a}$ are $3 \times 1$ vectors representing information related to each of the orthogonal leads. For simplicity, it is assumed that $s(n)$ is modeled by a step change,

$$
s(n)= \begin{cases}\frac{1}{\sqrt{D}}, & n=0, \ldots, \frac{D}{2}-1 \\ -\frac{1}{\sqrt{D}}, & n=\frac{D}{2}, \ldots, D-1 \\ 0, & \text { otherwise }\end{cases}
$$

where length of $s(n)$ is defined by the even-valued integer $D$. The length $M$ is chosen such that $s(n)$ is completely contained in the observation interval. The discrete-valued occurrence time $\theta$ is assumed to have a uniform a priori probability density function (pdf) such that

$$
p(\theta)= \begin{cases}\frac{1}{D}, & \theta=0, \ldots, D-1 \\ 0, & \text { otherwise. }\end{cases}
$$

A BPC is manifested in $\varphi(n)$ as a transition from a lower to a higher level with equal probability as a transition from a higher to a lower level. Therefore, the amplitude $a_{l}$ is in each lead characterized by a "two-point" a priori pdf

$$
p\left(a_{l}\right)= \begin{cases}\frac{1}{2}, & a_{l}=a_{l}^{0} \\ \frac{1}{2}, & a_{l}=-a_{l}^{0}\end{cases}
$$

where $a_{l}^{0}$ is a positive-valued constant representing the magnitude of the BPC. Hence, the magnitude of a transition in the $l$ th lead has a fixed size equal to $2 a_{l}^{0}$. The eight possible combinations of the amplitudes a that can occur are denoted by the set $\Omega_{a}$. The additive noise $\mathbf{w}(n)$ is assumed to be white and Gaussian with mean $m_{l}$ and variance $\sigma_{w_{l}}^{2}$. All random variables in $\theta$, $\mathbf{a}$ and $\mathbf{w}(n)$ are assumed to be mutually independent.

A Bayesian approach is considered for developing a BPC detector and takes its starting point in the binary detection problem with random, unwanted parameters, i.e., $\theta$ and a [17], [18]. The two hypotheses are characterized by the pdfs $p\left(\boldsymbol{\Phi}\left(n_{0}\right)\right.$ | $\left.\theta, \mathbf{a}, \mathcal{H}_{1}\right)$, describing that a BPC has occurred and $p\left(\boldsymbol{\Phi}\left(n_{0}\right)\right.$ $\left.\mathcal{H}_{0}\right)$, describing that nothing has changed; the matrix $\Phi\left(n_{0}\right)=$ $\left[\varphi\left(n_{0}\right) \ldots \varphi\left(n_{0}+M-1\right)\right]^{T}$ contains all observations of the three leads. The Bayesian detector requires that the likelihood 
ratio is tested against a threshold such that the hypothesis $\mathcal{H}_{1}$ is decided if

$$
\begin{aligned}
\Lambda\left(\boldsymbol{\Phi}\left(n_{0}\right)\right) & \stackrel{\operatorname{def} p\left(\boldsymbol{\Phi}\left(n_{0}\right) \mid \mathcal{H}_{1}\right)}{p\left(\boldsymbol{\Phi}\left(n_{0}\right) \mid \mathcal{H}_{0}\right)} \\
& =\frac{\sum_{\Omega_{a}} \sum_{\theta} p\left(\mathbf{\Phi}\left(n_{0}\right) \mid \theta, \mathbf{a}, \mathcal{H}_{1}\right) p(\theta, \mathbf{a})}{p\left(\mathbf{\Phi}\left(n_{0}\right) \mid \mathcal{H}_{0}\right)}>\text { threshold }
\end{aligned}
$$

where the two sums are introduced due to that the random variables $\theta$ and a are discrete-valued. The threshold is usually treated as a design parameter and selected such that a certain performance is achieved, e.g., in terms of false alarm rate. The detector given in (11) is rather demanding computationally and, therefore, a simplified detector based on the Taylor series expansion is derived (the derivation is presented in [19]). Assuming that the three leads have identical statistical properties, the simplified detector is defined by the sum of the energy of the matched filter output $y_{l}\left(n_{0}+\theta\right)$ of all leads and yields the following test against a threshold $\eta$ related to the previous threshold [19]

$$
\sum_{\theta=0}^{D-1} \sum_{l=1}^{3} y_{l}^{2}\left(n_{0}+\theta\right) \geq \eta .
$$

In order to accomplish detection of multiple BPCs, the detection test is repeated for successive values of $n_{0}$ until the entire signal has been processed. Hence, the detector performs a "sliding" hypothesis test to find out whether a BPC has occurred or not.

Two other modifications are introduced in the detector structure in order to improve performance. First, a nonlinearity is inserted after the matched filter in order to zero out any sample values below a certain threshold $\beta$; samples exceeding $\beta$ are unaffected. The purpose of this nonlinearity is to reduce the number of false detections due to noise. Second, a "refractory period" disqualifies detections made within a small time interval following the most recent detection; the time period is chosen to $10 \mathrm{~s}$. The inclusion of such a period reduces the risk that the onset and end of a single BPC is detected as two separate events.

\section{F. Noise Stage}

An extra postprocessing stage was considered after analysing the results, which can be added to the detection scheme (Fig. 2). The idea consists of making use of the information contained in the noisy beats which are rejected from the study. From the analysis of BPC recordings, it was found that one may generalize that a BPC episode implies a few noisy beats around its position (a noisy beat is a low SNR beat or a beat with high baseline wandering as defined in the preprocessing). We use the reverse (negative) implication to reject potential false BPC detections in the next way: no noisy beats found imply no BPC present, or at least, no need to detect a BPC (because the BPC would probably induce small ECG changes). To apply this rule it is required that a potential BPC at the Bayesian detector output must present a minimum number of noisy beats in the neighborhood (five noisy beats present in the surrounding 20 beats interval), to be considered as a BPC event.
TABLE III

TIME DELAYS ASSOCIATED WITH DIFFERENT PROCESSING STEPS OF THE BPC DETECTOR

\begin{tabular}{cc}
\hline Algorithm part & Introduced delay \\
\hline VCG angle series / KLT distance trends & $\approx 0 / 1 \mathrm{~s}$ \\
SNR beat rejection & $0 \mathrm{~s}$ \\
MAD beat rejection & $5 \mathrm{~s}$ \\
Trend resampling & $0 \mathrm{~s}$ \\
Detector & $0-7.5 \mathrm{~s}$ \\
\hline Total & $<\mathbf{1 2 . 5} \mathbf{~ s ~} / \mathbf{1 3 . 5} \mathbf{~ s}$
\end{tabular}

\section{G. Detection Delay}

The delay introduced by the different steps of the detector constitutes an important factor to know, in order to make the BPC detector to work properly together with the ischemia detector. A delay of 10-15 s may be an acceptable limit value in the BPC detector in order to not reduce the performance of the ischemia detector. The different parts of the algorithm introduce various delays, see Table III. The delay caused by the loop alignment is negligible since each estimate is calculated on a beat-by-beat basis. The delay introduced in the KLT method is less than $1 \mathrm{~s}$ (considering the convergence time of the adaptive algorithm). The SNR rejection operates on a beat series and does not introduce any delay. For the MAD rejection method it is necessary to introduce a delay to calculate the median absolute deviation (approximately of $5 \mathrm{~s}$ ). Resampling of the trends is done with a linear interpolation filter which introduces a negligible delay. The matched filter in the detector introduces a delay of maximum $5 \mathrm{~s}$. However, the matched filter and the remaining parts of the detector work in parallel, causing the detector delay to vary from 0 to $7.5 \mathrm{~s}$, depending on how large response from the trends the matched filter yields.

\section{Results}

Detector performance is assessed by measures which are database specific. Due to the differing characteristics of the two databases, different performance measures are considered. For the BPC database, the classical detection measures used e.g., for assessing communication systems, detection probability, $P_{D}$ and false alarm probability, $P_{F}$, are considered ${ }^{1}$. These measures can be defined in terms of the number of true detections $\left(N_{T}\right)$, false alarms $\left(N_{F}\right)$, and missed detections $\left(N_{M}\right)$

$$
\begin{aligned}
& P_{D}=\frac{N_{T}}{N_{T}+N_{M}} \\
& P_{F}=\frac{N_{F}}{N_{T}+N_{F}} .
\end{aligned}
$$

A different measure is used when evaluating the STAFF III database. Since this database does not contain any BPCs, the information is described by the false alarm rate, $R_{F}$, which is presented as the number of false BPCs per hour.

The performances of the two methods (without and with application of the extra noise stage) are shown in Table IV. The VCG angles presented slightly higher performance results than

\footnotetext{
${ }^{1}$ The notation in (13) and (14) is essentially the same as the ones used in some other studies i.e., sensitivity, $S$ and positive predictivity, $P^{+}$[20]. The relation between the two sets is $P_{D}=S$ and $P_{F}=1-P^{+}$, respectively.
} 
TABLE IV

Performance Statistics $(\mu \pm \sigma)$ FOR THE BPC Detectors on the BPC AND STAFF III Databases

\begin{tabular}{c||c|c|c|c}
\hline \multicolumn{1}{c||}{\multirow{2}{*}{ Detector }} & \multicolumn{2}{c|}{ BPC } & \multicolumn{2}{c}{ STAFF III } \\
\cline { 2 - 5 } & $P_{D}(\mu \pm \sigma)$ & $P_{F}(\mu \pm \sigma)$ & $R_{F}(c)(\mu \pm \sigma)$ & $R_{F}(a)(\mu \pm \sigma)$ \\
\hline \hline VCG angle series & $95 \% \pm 7$ & $3 \% \pm 5$ & $2 h^{-1} \pm 6$ & $7 h^{-1} \pm 14$ \\
\hline KLT distance trends & $89 \% \pm 13$ & $3 \% \pm 4$ & $4 h^{-1} \pm 13$ & $11 h^{-1} \pm 14$ \\
\hline \hline VCG + noise stage & $92 \% \pm 9$ & $1 \% \pm 2$ & $1 h^{-1} \pm 4$ & $3 h^{-1} \pm 9$ \\
\hline KLT + noise stage & $90 \% \pm 11$ & $1 \% \pm 3$ & $1 h^{-1} \pm 3$ & $2 h^{-1} \pm 7$ \\
\hline
\end{tabular}
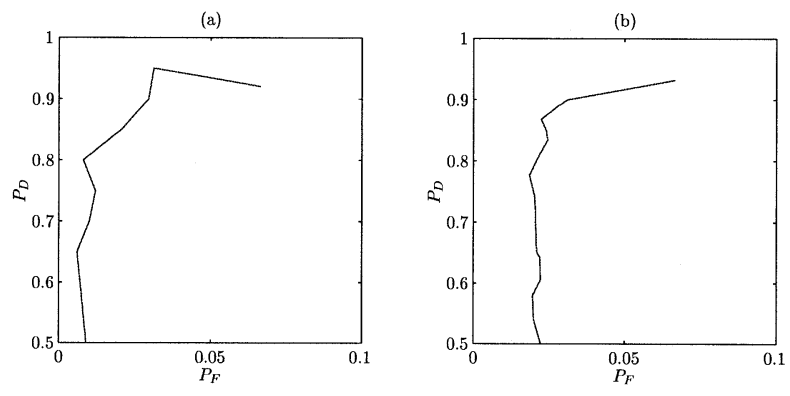

Fig. 6. ROC graphs for the VCG angle series (a) and KLT coefficients (b) varying $\eta$ with a fixed $\beta$ amplitude, on the BPC database.

did the KLT distance, especially regarding detection probability on the BPC database and false alarm rate on the STAFF III database. When the extra noise stage is applied both methods reach similar performances. In general, for both methods (in the two presented versions, i.e., considering the extra noise stage or not), $P_{F}$ values are small $(\leq 3 \%)$. The obtained $P_{D}$ values are also quite high, ranging from $89 \%$ to $95 \%$. Finally, $R_{F}$ values range from 2 to $11 \mathrm{~h}^{-1}$ and from 1 to $3 \mathrm{~h}^{-1}$ when the extra noise stage is considered. The false alarm rate in angioplasty-induced ischemia recordings, $R_{F}(a)$, is approximately three times that of the control recordings, $R_{F}(c)$. The standard deviation of the methods is relatively large between subjects as it can be deduced from the results.

The receiver operating characteristics (ROC) constitute a common approach to present detector performance. The ROC display the relationship between $P_{D}$ and $P_{F}$ for different values of a certain detector parameter. The ROC of the BPC database were calculated choosing as parameter for evaluation that judged as the most critical for performance: $\eta$ threshold. In Fig. 6(a) and (b), the ROC curves for the VCG angles series and KLT trends, respectively, are plotted for different values of $\eta$, starting with the largest parameter value in the lower left corner. These curves were calculated for both detectors without the extra noise stage. Similar curves are obtained when this stage is applied. In order to investigate the STAFF III database (control and angioplasty recordings), the $\eta$ parameter was again considered and the results are presented in terms of false alarm rate, $R_{F}$, in Fig. 7 . It can be seen that the $R_{F}$ parameter is less sensitive to variations in the detector parameter in control recordings $\left(R_{F}(c)\right)$ than in angioplasty recordings $\left(R_{F}(a)\right)$.

\section{Discussion AND CONCLUSION}

Several parameters control the performance of the proposed methods to detect BPCs. A tradeoff among $P_{D}, P_{F}$, and $R_{F}$ needs to be achieved. The $P_{D}$ parameter reflects true BPCs missed by the BPC detector and, therefore, how many false ischemic alarms will be activated by the BPCs during monitoring. It is also important to minimize $P_{F}$ in order to avoid cancellation of events during monitoring due to sources different from BPCs. Finally, the $R_{F}$ parameter needs to be as low as possible (especially in angioplasty recordings, $R_{F}(a)$ ) because an ischemic event confused as a BPC would be ignored and the ischemia alarm would be cancelled. From the perspective of signal processing and BPC detection the goal is to get high $P_{D}$ and low $P_{F}$ values. However, from the clinical perspective, it should be more important to decrease $R_{F}$ (mainly $R_{F}(a)$ ), thus avoiding that a true ischemic episode could be cancelled. Therefore, future research should be addressed mainly on these aspects to tune the parameters detector.

Although the loop alignment performed slightly better on the databases, the difference is so marginal that it can be concluded that the two, distinctly different, approaches lead to the same result. The KLT method includes more information from the ECG (considering both QRS and ST-T complexes). However, in practice, results show that maybe KLT series are affected in a longer time period by each BPC as a consequence of using both complexes and the adaptive estimation. Introducing in parallel the noise criterion stage both methods work essentially equal.

One aspect that should not be forgotten is that in the STAFF III database recordings the induced ischemia comes from a sudden occlusion and, therefore, changes take place in a shorter period of time than during a common transient ischemic event and they probably also have greater ischemic magnitude. This means that the time and amplitude signature for these ischemic events is more likely to that of BPCs and thus more difficult to distinguish. It is also noticeable that each STAFF III angioplasty recording ( $a$ recordings) includes one ischemic episode which lasts around 5 min, thus leading in a STAFF III recording to 12 events per hour, a rate that is much higher than that usually expected in ambulatory recordings. This reasoning yields that $R_{F}$ results during ambulatory ischemia monitoring (not PTCA ECGs) would probably be lower than the ones obtained in this work, which can be relatively high (consider that $R_{F}(a)=2$, with 12 events per hour, means that one-sixth of the PTCA ischemic episodes are misclassified as BPCs).

Our group has been previously involved in the study of ischemia detectors with the aim to design detection schemes which consider global or morphology changes on the ECG complexes under different approaches [21], [22]. We tested the proposed ischemia detector based on the root mean square (RMS) difference series of ECG intervals [22] on the BPC database. The false detections for ST-T complex obtained with 
(a)

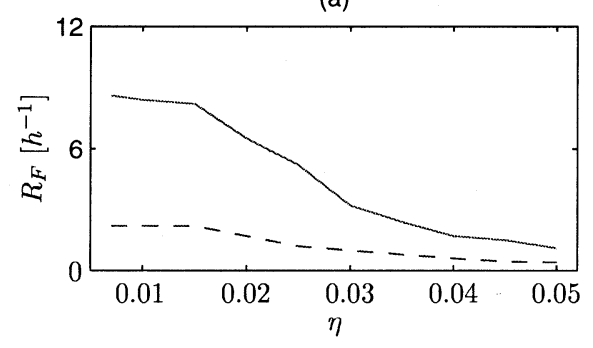

(b)

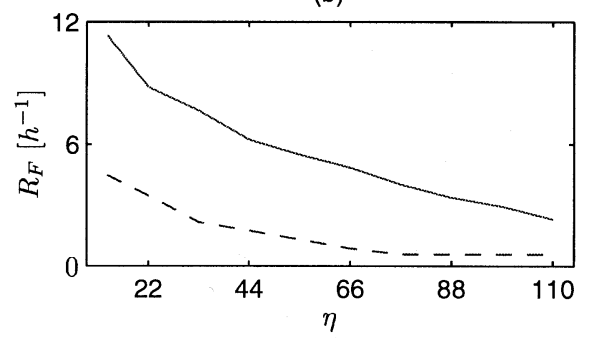

Fig. 7. VCG angle series (a) and KLT trends (b) performance on the STAFF III database: $R_{F}(a)$ (solid line) and $R_{F}(c)$ (dashed line) are plotted varying $\eta$.

this ischemia detector are less than $2 \%$ of total BPCs, thus making it less necessary the use of a BPC detector working in parallel with it. However, by using classical ischemia detectors the false alarm rate is much higher (43\%) as it has been shown in the description of the BPC database and the application of a BPC detector would be very useful. A third approach to the BPC detection problem could be to use the RMS series as input information to characterize BPCs (in the same way as KLT or VCG angle trends). Thus, the same scheme could be used both for ischemia and BPC detection. However the results obtained are a bit less satisfactory than with the two presented methods: $P_{D}=88 \%, P_{F}=6 \%, R_{F}(c)=2 \mathrm{~h}^{-1}$, and $R_{F}(a)=3 \mathrm{~h}^{-1}$ (obtained using the extra noise stage). An explanation to the fact that KLT trends obtain better results than RMS series in BPC detection but worse in ischemia detection may come from the SNR trend values. In general, KLT trends are much cleaner than RMS series (first KLT coefficients recover main signal features filtering high frequency noise content) and RMS series, especially during a BPC, present a high noise content. Then many beats can be rejected from the RMS series, making it difficult to detect the corresponding BPC.

An interesting extension of this work would be the test of the proposed methods in a database simultaneously containing both ischemia events and BPCs. The collection of such a database is however associated with considerable efforts and costs.

\section{ACKNOWLEDGMENT}

The authors would like to thank Prof. O. Pahlm, the Department of Clinical Physiology, Lund University, for his valuable discussion and for making the acquisition of the BPC database possible. This study is part of the STAFF Studies.

\section{REFERENCES}

[1] M. Adams and B. Drew, "Body position effects on the electrocardiogram: Implications for ischemia monitoring," J. Electrocardiol., vol. 30, no. 4, pp. 285-291, 1997.

[2] T. Jernberg, B. Lindahl, M. Högberg, and L. Wallentin, "Effects on QRSwaveforms and ST-T-segment by changes in body position during continuous 12-lead ECG: A preliminary report," in Computers in Cardiology. Los Alamitos, CA: IEEE Comp. Soc., 1997, pp. 461-464.

[3] B. Nørgaard, B. Rasmussen, M. Dellborg, and K. Thygesen, "Positional changes of spatial QRS- and ST-segment variables in normal subjects: Implications for continuous vectorcardiography monitoring during myocardial ischemia," J. Electrocardiol., vol. 33, no. 1, pp. 23-30, 2000.

[4] S. Nelwan, S. Meij, T. vanDam, and J. Kors, "Correction of ECG variations caused by body position changes and electrode placement during ST-T monitoring," J. Electrocardiol., vol. 34 Suppl:1, no. 4, 2001.
[5] F. Jager, R. Mark, G. Moody, and S. Divjak, "Analysis of transient ST segment changes during ambulatory monitoring using the KarhunenLoève transform," in Computers in Cardiology. Los Alamitos, CA: IEEE Comp. Soc., 1992, pp. 691-694.

[6] A. Taddei, G. Distante, M. Emdin, P. Pisani, G. B. Moody, C. Zeelenberg, and C. Marchesi, "The European ST-T database: Standards for evaluating systems for the analysis of ST-T changes in ambulatory electrocardiography," Eur. Heart J., vol. 13, pp. 1164-1172, 1992.

[7] J. García, P. Lander, L. Sörnmo, S. Olmos, G. Wagner, and P. Laguna, "Comparative study of local and Karhunen-Loeve based ST-T indexes in recordings from human subjects with induced myocardial ischemia," Comput. Biomed. Res., vol. 31, pp. 271-292, August 1998.

[8] J. Pettersson, E. Carro, L. Edenbrandt, O. Pahlm, M. Ringborn, L. Sörnmo, and G. W. S. Warren, "Changes in high frequency QRS components are more sensitive than ST segment deviations for detecting acute coronary artery occlusion," J. Amer. College Cardiol., vol. 36, pp. $1827-1834,2000$.

[9] L. Edenbrandt and O. Pahlm, "Vectorcardiogram synthesized from a 12-lead ECG: Superiority of the inverse dower matrix," J. Electrocardiol., vol. 21, pp. 361-367, 1988.

[10] L. Sörnmo, "Time-varying filtering for removal of baseline wander in exercise ECG's," in Computers in Cardiology. Los Alamitos, CA: IEEE Comput. Soc. Press, 1991, pp. 145-148.

[11] L. Sörnmo, O. Pahlm, and M. E. Nytgårds, "Adaptive QRS detection: A study of performance," IEEE Trans. Biomed. Eng., vol. BME-32, pp. $392-401,1985$.

[12] L. Sörnmo, "Vectorcardiographic loop alignment and morphologic beat-to-beat variability," IEEE Trans. Biomed. Eng., vol. 45, pp. 1401-1413, Dec. 1998.

[13] C. W. Therrien, Discrete Random Signals and Statistical Signal Processing, ser. Signal Processing. Englewood Cliffs, NJ: Prentice-Hall, 1992.

[14] P. Laguna, G. Moody, J. García, A. Goldberger, and R. Mark, "Analysis of the ST-T complex of the electrocardiogram using the KarhunenLoève transform: Adaptive monitoring and alternans detection," Med. Biol. Eng. Comput., vol. 37, pp. 175-189, 1999.

[15] J. García, G. Wagner, L. Sörnmo, S. Olmos, P. Lander, and P. Languna, "Temporal evolution of traditional vs. transformed ECG-based indexes in patients with induced myocardial ischemia," J. Electrocardiol., vol. 33, no. 1, pp. 37-47, 2000.

[16] F. Hampel, E. Ronchetti, P. Rousseeuw, and W. Stahel, Robust Statistics, ser. Probability and Mathematical Statistics. New York: Wiley, 1986.

[17] H. van Trees, Detection, Estimation and Modulation Theory. New York: Wiley, 1968-1971, vol. I-III.

[18] S. M. Kay, Fundamentals of Statistical Signal Processing: Detection Theory, 2nd ed, ser. Signal Processing. Upper Saddle River, NJ: Prentice Hall, 1998, vol. II.

[19] M.Åström, J. García, P. Laguna, O. Pahlm, and L. Sörnmo, "Detection of Body Position Changes Using the Surface ECG," Med., Biol. Eng., Comput., vol. 41, pp. 164-171, 2003.

[20] F. Jager, G. B. Moody, A. Taddei, and R. G. Mark, "Performance measures for algorithms to detect transient ischemic STsegment changes," in Computers in Cardiology. Los Alamitos, CA: IEEE Comput. Soc. Press, 1991, pp. 369-372.

[21] J. García and P. Laguna, "Automatic ischemia episodes detection on ECG recordings," in Proc. 5th Conf. Eur. Soc. Eng. and Med., 1999, pp. 387-388.

[22] J. García, L. Sörnmo, S. Olmos, and P. Laguna, "Automatic detection of ST-T complex changes on the ECG using filtered RMS difference series: Application to ambulatory ischemia monitoring," IEEE Trans. Biomed. Eng., vol. 47, pp. 1195-1201, Sept. 2000. 


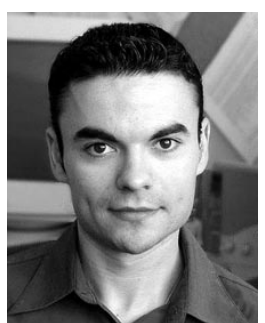

José García was born in Zaragoza, Spain in 1971. He received the M.S. degree is physics and the Ph.D. degree from the University of Zaragoza (U.Z.), Zaragoza, Spain, in 1994 and 1998, respectively.

He worked during 1999 as a Postdoctoral Researcher with the Department of Electronics Engineering and Communications in the Polytechnic Center at U.Z where he is now an Associate Professor in Telematics Engineering area. His research interests are in telemedicine, biomedical signal processing, and other related topics.

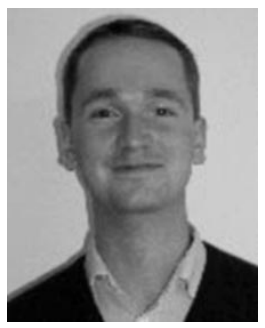

Magnus Åström completed the master's thesis at the Department of Applied Electronics (now the Department of Electroscience), Lund University, Lund, Sweden, in the field of medical signal processing in 1997. After finishing the M.Sc. he continued at the department as a Ph.D. degree student within the Signal Processing Group. In 2000, he received the Licentiate in Engineering degree in signal processing.

$\mathrm{He}$ is focusing on methods for detecting myocarvelopment for pacemakers. dial ischemia using ECG signals and algorithm de-

Javier Mendive completed the master's degree thesis at the Department of Electronics Engineering and Communications, Polytechnic Center, University of Zaragoza (U.Z.), Zaragoza, Spain, in the field of biomedical signal processing in 2001 .

$\mathrm{He}$ is now working on communications infrastructure management.

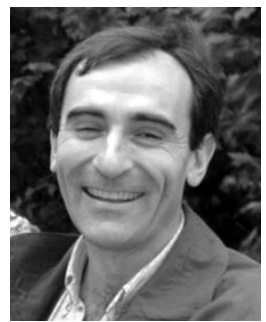

Pablo Laguna was born in Jaca (Huesca), Spain, in 1962. He received the M.S. degree in physics and the Ph.D. degree in physics science from the Science Faculty at the University of Zaragoza, Zaragoza, Spain, in 1985 and 1990, respectively. The Ph.D. thesis was developed at the Biomedical Engineering Division of the Institute of Cybernetics (U.P.C.-C.S.I.C.) under the direction of Pere Caminal.

He is an Associated Professor of Signal Processing and Communications with the Department of Electronics Engineering and Comunications at the Centro Politécnico Superior, University of Zaragoza. From 1987 to 1992 he worked as Assistant Professor of Automatic Control, Department of Control Engineering, Politecnic University of Catalonia (U.P.C.), Catalonia, Spain, and as a Researcher at the Biomedical Engineering Division of the Institute of Cybernetics (U.P.C.-C.S.I.C.). His professional research interests are in signal processing, in particular applied to biomedical applications.

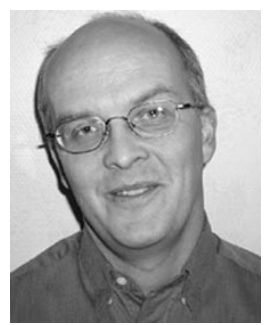

Leif Sörnmo (S'80-M'85) received the M.Sc. and D.Sc. degrees in electrical engineering from Lund University, Lund, Sweden, in 1978 and 1984, respectively.

He held a research position with the Department of Clinical Physiology, Lund University, from 1983 to 1995 , where he worked on computer-based ECG analysis. Since 1990, he has been with the Signal Processing Group, Department of Electroscience, Lund University, where he now holds a position as a Professor in biomedical signal processing. He was on the editorial board of Computers in Biomedical Research from 1997 to 2000. His main research interests include statistical signal processing and modeling of biomedical signals. His current research projects include high-resolution ECG analysis, methods in ischemia monitoring, time-frequency analysis of atrial fibrillation, power efficient signal processing in pacemakers, and detection of otoacoustic emissions

Dr. Sörnmo has been an Associate Editor of the IEEE TRANSACTIONS ON BIOMEDICAL ENGINEERING since 2001. 